\title{
Age, Physical Activity Motivation and Perceived Stress in Minority Girls
}

\author{
Amber R. Cordola Hsu (D) 1, Selena T. Nguyen-Rodriguez (D) 2 , and Donna Spruijt-Metz (D) 3 \\ ${ }^{1}$ Claremont Graduate University, School of Community \& Global Health \\ ${ }^{2}$ California State University, Long Beach, Department of Health Science \\ ${ }^{3}$ University of Southern California, Institute for Health Promotion \& Disease Prevention \\ Research, Division of Health Behavior, Departments of Preventive Medicine, USC mHealth \\ Collaboratory, Center for Economic and Social Research, Department of Psychology
}

\begin{abstract}
Background: Physical activity in childhood and adolescence helps support physical and emotional health. Purpose: The study aimed to investigate if age was related to motivation for physical activity in minority girls, and whether the relationship may be potentially mediated by psychological or physiological stress. Methods: This cross-sectional observational study recruited Latino and African American girls ages 8 12 years $(n=79)$ in Tanner stage 1 or 2 via purposive sampling. Intrinsic motivation and perceived stress were measured by self-report survey; morning salivary cortisol samples were taken to calculate cortisol awakening response to estimate biological stress reactivity. Results: Increased age was related to higher intrinsic motivation to engage in physical activity. Lower perceived stress and lower awakening cortisol response were associated with higher intrinsic motivation. Bootstrapped mediation results indicated perceived stress may be a pathway through which age impacts intrinsic motivation for physical activity. Conclusion: While motivation to engage in physical activity may increase with age, perceived stress may dampen this motivation, resulting in decreased physical activity. Interventions to help increase preadolescent girls' engagement in active behaviors may benefit from reducing children's perceptions of stress.
\end{abstract}

(C) 2019 and CC-BY 4.0 licensed by the authors.

Keywords: Age, Perceived stress, Cortisol, Intrinsic motivation, Minority girls

\section{Introduction}

Physical activity in childhood and adolescence improves strength and endurance, helps build healthy bones and muscles, helps control weight, and may improve blood pressure and cholesterol levels (U.S. Department of Health and Human Services, 2018). According to the CDC Morbidity Mortality Weekly Report (MMWR) Youth Risk Behavior Surveillance System (2018), 19.5\% of females in grades 9-12 failed to participate in at least $60 \mathrm{~min}$ of physical activity on at least one day; this lack of participation in physical activity was higher among black $(26.6 \%)$ and Hispanic $(20.0 \%)$ females. Participation in physical activity also declines as young people age (Spruijt-Metz et al., 2013). Further, adolescent girls report lower levels of physical activity than boys (Belcher et al., 2010) and display greater rates of decline in physical activity across adolescence (National Center for Health Statistics, 2017).

\section{Self-Determination Theory}

Self-Determination Theory (SDT) presents a broad framework for the study of human motivation (Deci \& Ryan, 1985). This theory describes the continuum of motivation ranging from being completely unmotivated (amotivation) to externally motivated (extrinsic motivation) through being completely selfmotivated (intrinsic motivation). SDT posits that self-determined intrinsic motivation is key to supporting healthy behaviors based on the satisfaction of behaving "for its own sake" (Ryan \& Deci, 2000). Research shows that adolescents with a high motivation profile (intrinsic and extrinsic) were more physically active to a greater degree than those with a low motivation profile (Yli-Piipari, Watt, \& Jaakkola, 2009). In addition, Owen, Smith, and Lubans (2014) 
performed a meta-analysis that showed forty-six studies reported overall levels of self-determined motivation had weak to moderate, positive associations with physical activity $(\rho=0.21$ 0.31 , whereas, intrinsic motivation and identified regulation (i.e., somewhat internal motivation) had moderate, positive associations with physical activity $(\rho=0.27-0.38)$ (Owen et al., 2014). Thus, identification of factors that impact intrinsic motivation may elucidate strategies to ultimately increase activity levels. Despite the popularity of motivation research, investigations examining the impact of stress on intrinsic motivation are lacking, particularly in minority girls.

\section{Age and Gender-related Factors of Physical Activity}

According to Tremblay and Frigon (2005), an age-related factor leading to low physical activity among girls may be the psychological experience of puberty and in particular the timing of pubertal maturation. During the adolescent years, the intensity and frequency of parent-child conflict increases, and parent-child interactions are marked by more negative emotions (e.g., Shanahan, McHale, Osgood, \& Crouter, 2007). Female adolescents tend to respond more negatively to stress than their male counterparts, which may be due to emerging differences in the in physiological response systems, such as the hypothalamic-pituitary-adrenal (HPA) system (Ordaz \& Luna, 2012). The multiple changes at the age when girls enter puberty are likely a significant source of stress for girls. Motivation to engage in healthy behaviors can be impacted by reactions to stress (Glanz \& Schwartz, 2008), such as physical activity/exercise. One study found that stress reduced motivation for physical activity among active adults but increased motivation for inactive adults (Lippke, Wienert, Kuhlmann, Fink, \& Hambrecht, 2015). This underscores the utility of exploring the influence of age-related stress on determinants of physical activity, such as motivation, to better understand why girls become less active as they get older.

\section{The Cortisol Awakening Response}

Cortisol is a glucocorticoid hormone recognized as a primary marker of the biological stress response (Fries, Dettenborn, \& Kirschbaum,
2009) and biomarker of psychological stress (Lam, 2012), where elevated cortisol levels are one result from stress experienced in a person's environment via the HPA axis (Cohen, JanickiDeverts, \& Miller, 2007). The HPA axis acts to maintain homeostasis in reaction to physical and environmental challenges (Tsigos \& Chrousos, 2002), such as stressors, triggering release of hormones from the adrenal cortex to address those challenges, with cortisol being the primary glucocorticoid secreted in humans (Carrasco \& Van de Kar, 2003). In general, cortisol patterns follow a diurnal pattern where levels peak in the early morning then steadily decrease across the day, reaching the nadir in the earlier half of the night (Tsigos \& Chrousos, 2002); the cortisol awakening response is defined by a sharp increase in cortisol that occurs within 20-30 minutes of awakening (Wilhelm, Born, Kudielka, Schlotz, \& Wüst, 2007).

Researchers have posited that the cortisol awakening response is an indicator of anticipated daily burden (Fries, Dettenborn, \& Kirschbaum, 2009) as well as biological stress reactivity (Sanchez, Urizar, \& Yim, 2019). Research indicates that chronic stress may lead to increased cortisol awakening response, where perceived stress appears to further increase the awakening cortisol response (Wüst, Federenko, Hellhammer, \& Kirschbaum, 2000). The cortisol awakening response is of particular interest to physical activity research, as it has been theorized that it plays a role in harnessing energy to prepare for daily activities (Kudielka \& Wüst, 2010). Pubertal development is a time when stressors increase the arousal or reactivity properties of the HPA and hypothalamic pituitary gonadal (HPG) axis hormones, therefore, increasing the potential for these HPA- and HPG-hormones to have an effect on adolescent emotions and behaviors (Shirtcliff \& Ruttle, 2010). Given scarce research on the topic, examining whether increased perceived stress brought on at the age of pubertal onset may negatively impact motivation to engage in physical activity presents a novel avenue of investigation.

\section{The Biopsychosocial Model of Health}

The biopsychosocial model of health (Engel, 1977; Borrell-Carrió, Suchman, \& Epstein, 2004) 
guides the current investigation as it provides a framework to explain how biological, psychological, behavioral and social factors interact to impact health. This approach recognizes that health and illness are not solely the result of biological processes, but that complex (e.g., multidimensional, synergistic and bidirectional) interactions with psychological, behavioral and social processes lead to differences in health outcomes. In the current analysis, we explore age as a biological marker that may impact motivation for physical activity (psychosocial factor that impacts behavior), where stress may increase at the age of pubertal onset due to both biological processes (e.g., activation of HPA axis) and social influences. We examine whether stress may be a potential underlying mechanism in this age-motivation association using a biological (cortisol) and psychological (perceived stress) indicator.

\section{The Present Study}

Given extant literature and the multifaceted mechanisms of influence posited in the biopsychosocial model (Engel, 1977; BorrellCarrió et al., 2004), it is plausible that stress, both psychological and physiological, may play a role in the age-related decline in physical activity, possibly by impacting motivation to engage in physical activity. Considering the psychosocial and physiological changes that occur during the transition from late childhood to early adolescence, the current exploratory analyses tested whether age was related to intrinsic motivation for physical activity in a sample of Latino and African American prepubertal girls, and whether age may indirectly impact motivation through perceived stress and awakening cortisol response. Since the objective of the current exploration was to identify factors related to motivation for physical activity, and the sample was too small to conduct path analyses, physical activity was not investigated as part of the main analysis. Nonetheless, results of associations between motivation and physical activity are reported.

\section{Methods}

\section{Study Design}

This cross-sectional observational study uses data derived from a clinical data collection visit.

\section{Participants}

The data $(n=79)$ used for the current study are from a larger biobehavioral study of physical activity among minority girls; the sample is comprised of those who completed the baseline visit. Participants were pre-pubertal Latino and African American girls (Spruijt-Metz et al., 2013), recruited via purposive sampling. Inclusion criteria were as follows: 1) Female gender; 2) African American or Latino ethnicity (based on self-report that child, both parents, and all four grandparents were African American or Latino); 3) Tanner stage 1 or 2 (Tanner stage estimates pubertal stage, based on ratings of secondary sex characteristics of children across puberty (Emmanuel \& Bokor, 2019)); and 4) Age 8-12 years. Exclusion criteria were: 1) Children with symptoms of polyuria, polydipsia with or without unexplained weight loss, or a fasting plasma glucose > $126 \mathrm{mg} / \mathrm{dl}$; 2) taking any medications known to influence body composition or insulin action or insulin secretion; 3) diagnosed with syndromes or diseases that may influence insulin action and secretion or body composition and fat distribution; or 4) previously diagnosed with any major illness since birth.

\section{Measures}

Age. Participants age was calculated in months, by subtracting their date of birth from the date of assessment.

Intrinsic Motivation. Motivation for exercise was measured via the Exercise Self-Regulation Questionnaire (SRQ-E) (Levesque, Williams, \& Elliot, 2007), made up of 16 items, with a 7-point Likert scale response option ranging from "not at all true" to "very true." This scale assesses the reasons a person engages in regular exercise and provides information about external regulation, introjected regulation, identified regulation, and intrinsic motivation, resulting in a measure of one's autonomy regarding exercise/physical 
activity. For example, "The reason I would exercise regularly is: Because I feel that I want to take responsibility for my own health." (Levesque et al., 2007). Four items make up the intrinsic motivation subscale; one item seemed to be conceptually different from the items (internal consistency reliability was lower when the item was included), therefore it was not included in the final scale score. Scores were derived by taking the mean of the remaining three items $(\alpha=.648)$. This scale has not been validated for use in minority children, although it has been previously shown to have good psychometric quality in youth samples (Foran, Cermak, \& Spruijt-Metz, 2013; Goudas, Biddle, \& Fox, 1994; SpruijtMetz, Nguyen-Michel, Goran, Chou, \& Huang, 2008; Wang, \& Biddle, 2001).

Perceived Stress. Perceived Stress was assessed via the Perceived Stress Scale (PSS) (Cohen, Kamarck, \& Mermelstein, 1983). This measure is intended to assess perception of stress in the last month. Developed from data on a community sample, the PSS has good psychometric properties. However, the scale has not been validated with youth populations (Lee, 2012). In a previous project with minority middle school students (Spruijt-Metz, Nguyen-Michel, Goran, Chou, \& Huang, 2008; Nguyen-Rodriguez, Unger, \& Spruijt-Metz, 2009), short interviews with adolescents were conducted to gauge suitability of the measure for these samples. The qualitative findings resulted in the addition of 3items to the original scale (felt had too much work to do; had to keep secrets; worried about social life), increasing face validity for the target population. This modified 17-item version of this scale was employed (the addition of these items increased internal consistency reliability for this measure). Responses were provided across a 5point Likert scale, ranging from Never $(0)$ to Very Often (4). For example, "1. In the last month, how often have you been upset because of something that happened unexpectedly?" (Cohen et al., 1983). Seven items were reversed scored so that higher scores would indicate higher perceived stress. Scores were calculated by taking the sum of items (Cronbach $\alpha=.681$ ).

Physiological Stress Reactivity. Cortisol awakening response was used to indicate physiological stress reactivity; it was calculated by subtracting cortisol values at 30 minutes after awakening from awakening cortisol values. Salivary cortisol samples were taken at the overnight visit at the GCRC. Samples were collected by nurses, following a standardized protocol. Participants provided saliva samples with salivettes (Sarstedt, Newton, NC) at 10pm, upon awakening, then 15 minutes and 30 minutes after awakening. The salivettes were placed for two minutes in the child's inner cheek and transferred to plastic vials. Samples were centrifuged at 2,500 rpm for $10 \mathrm{~min}$, and saliva supernatant was stored in freezers $\left(-70{ }^{\circ} \mathrm{C}\right)$ to allow for subsequent assaying. At the GCRC lab, cortisol was assayed with an automated enzyme immunoassay (Tosoh AIA 600 II analyzer, Tosoh Bioscience, South San Francisco, CA). The assay sensitivity was $0.02 \mu \mathrm{g} / \mathrm{dl}$; the coefficient of variation for inter-assays was $11.2 \%$ and $8.2 \%$ for intra-assay. All samples were assayed at the same time.

Physical Activity. Physical activity was measured by a uniaxial Actigraph GT1M accelerometer programmed to collect data in 15second epochs, worn on the right hip with an elastic band. Data were considered valid if worn for at least $10 \mathrm{~h}$ on 4 days. Data were converted to 60 -second epochs in order to process using an adapted version of the National Cancer Institute's National Health and Nutrition Examination Survey SAS code (available at: http://riskfactor.cancer.gov/ tools/nhanes_pam). The cut points for moderate (4 METs) and vigorous (7 METs) activity were adjusted for age using the criteria from Freedson, Melanson, and Sirard (1998). Time spent in each level of intensity were calculated by the total minutes spent within the given thresholds.

\section{Procedures}

Bilingual and diverse study team members recruited participants from local public areas, such as parks and local shops. School and community-based events, including PTA meetings and health fairs, were also used as recruitment locations. Parents were approached to ask if they would like to learn more about the study. If interested, parents were asked to answer screening questions to determine eligibility based 
on age and ethnicity. If potential participants met age and ethnicity criteria, a consent visit was scheduled. Written parental permission from both parents (unless one had sole guardianship) and child assent were required for participation in the study. All study procedures were approved by the University of Southern California's Institutional Review Board.

Detailed procedures of the parent study are described elsewhere (Spruijt-Metz et al., 2013); only those relevant to the current study are included here. Participants completed paper-andpencil surveys, including demographics and scales to assess various psychosocial factors and determinants of physical activity, including perceived stress and motivation for physical activity. Surveys were completed in the evening of an overnight visit at the General Clinical Research Center (GCRC); participants also received a physical exam by a physician assistant which included assessment of Tanner Stage. Physical activity was assessed by accelerometry for the seven days following the overnight visit.

\section{Analyses}

Descriptive statistics were derived from means and frequencies. Preliminary bivariate analyses (independent-samples t-tests) showed that potential confounders, ethnicity and pubertal stage, were not related to the mediators or outcome, therefore, to preserve statistical power, these and other covariates were not included in models. Primary analyses were run using the Preacher and Hayes (2008) method in order to test mediation with bias-corrected bootstrapping to obtain $95 \%$ confidence intervals for indirect effects (using 5000 bootstrap resamples); this procedure produces unstandardized beta coefficients.

Two separate models were run to independently assess the indirect effect of perceived stress vs. physiological stress (cortisol awakening response); since perceived stress and cortisol awakening response were not correlated in the current sample $(\mathrm{r}=.032, \mathrm{p}=.818)$, a multiple mediation model was not warranted. Thus, one model tested the direct effects of age on motivation for physical activity, adding perceived stress to assess its indirect effect. The second model assessed the age-motivation association, adding cortisol awakening response to explore whether it played a role in this relationship. Confidence intervals (CIs) allow for assessment of precision of the estimates and $95 \%$ CIs indicate non-significance if the interval included zero; effect sizes are reported to provide information on the practical significance of findings. Estimates of effect size included the ratio of the indirect effect to the total effect $\left(\mathrm{P}_{\mathrm{M}}\right)$ for mediation effects (Preacher \& Kelley, 2011) and an adjusted r-squared for the overall model. SPSS version 21 (Armonk, NY: IBM Corp) was used for all statistical analyses.

\section{Results}

Characteristics of the sample are reported in Table 1. The sample was of diverse ethnic background, with the majority being Latino. Participants were evenly split between Tanner Stages 1 and 2. On average, the sample was of normal weight, based on youth BMI percentile.

\section{Cortisol Awakening Response}

Analyses testing physiological stress as a possible mediator revealed a statistically significant positive relationship (total effect) between age and intrinsic motivation for physical activity $(B=$ $.0534, \mathrm{SE}=.0164, \mathrm{p}=.0019$; see Table 2). Age was not significantly related to cortisol awakening response $(\mathrm{p}=$.9289). Cortisol awakening response was negatively associated with intrinsic motivation for physical activity ( $\beta$ $=-0.6997, \mathrm{SE}=0.3033, \mathrm{p}=.0250)$. Bootstrap results (see Table 2) showed that the unique indirect effect through cortisol awakening response was not significant $(B=-0.0002, \mathrm{SE}=$ 0.0054, 95\% CI: -0.0106, 0.0114). Thus, although inclusion of awakening cortisol in the model slightly changed the beta and p-value from the total effect (direct effect of age on motivation, $\beta=0.0529, p=.0015$ ), results of bootstrap analyses illustrate this change is non-significant. These findings indicate that the relationship between age and intrinsic motivation for physical activity $\left(\mathrm{P}_{\mathrm{M}}=.0037\right)$ may not be affected by cortisol awakening response. 
Table 1.

Characteristics of the Study Sample $(\mathrm{N}=79)$

\begin{tabular}{|c|c|c|c|c|c|}
\hline & $\mathrm{N}$ & $\%$ & Mean & $\mathrm{SD}$ & Range \\
\hline Age (months) & & & 117.52 & 10.91 & $96-139$ \\
\hline \multicolumn{6}{|l|}{ Ethnicity* } \\
\hline African American & 24 & 30.4 & & & \\
\hline Hispanic/Latina & 55 & 69.6 & & & \\
\hline \multicolumn{6}{|l|}{ Female Tanner Stage* } \\
\hline Stage 1 & 39 & 49.4 & & & \\
\hline Stage 2 & 40 & 50.6 & & & \\
\hline BMI Percentile & & & 80.0 & 23.53 & $1.56-99.74$ \\
\hline Intrinsic Motivation Physical Activity & & & 5.56 & 1.47 & $1-7$ \\
\hline Cortisol Awakening Response & & & 0.79 & 0.56 & $-0.72-2.03$ \\
\hline Perceived Stress & & & 29.68 & 9.60 & $0-47$ \\
\hline \multicolumn{6}{|l|}{ Moderate Physical Activity } \\
\hline $\begin{array}{l}\text { Minutes of Physical Activity } \\
\text { (average per day) }\end{array}$ & & & 42.44 & 21.39 & $11.75-109.20$ \\
\hline $\begin{array}{l}\text { Percent Time Spent in Physical Activity } \\
\text { (average per day) }\end{array}$ & & & .05 & .02 & $.02-.13$ \\
\hline
\end{tabular}

\section{Cortisol Awakening Response}

Analyses testing physiological stress as a possible mediator revealed a statistically significant positive relationship (total effect) between age and intrinsic motivation for physical activity $(B=$ $.0534, \mathrm{SE}=.0164, \mathrm{p}=.0019$; see Table 2). Age was not significantly related to cortisol awakening response $(\mathrm{p}=$.9289). Cortisol awakening response was negatively associated with intrinsic motivation for physical activity ( $(3$ $=-0.6997, \mathrm{SE}=0.3033, \mathrm{p}=.0250)$. Bootstrap results (see Table 2) showed that the unique indirect effect through cortisol awakening response was not significant $(\beta=-0.0002, \mathrm{SE}=$ 0.0054 , 95\% CI: -0.0106, 0.0114). Thus, although inclusion of awakening cortisol in the model slightly changed the beta and p-value from the total effect (direct effect of age on motivation, $\beta=0.0529, \mathrm{p}=.0015$ ), results of bootstrap analyses illustrate this change is non-significant. These findings indicate that the relationship between age and intrinsic motivation for physical activity $\left(\mathrm{P}_{\mathrm{M}}=.0037\right)$ may not be affected by cortisol awakening response.

Table 2.

Indirect Effect of Cortisol Awakening Response in the Relationship between Age and Intrinsic Motivation for Physical Activity

\begin{tabular}{lrrrr}
\hline & \multicolumn{3}{c}{ Intrinsic Motivation for Physical Activity } \\
\cline { 2 - 5 } & B & SE & p-value & 95\% CI \\
\cline { 2 - 5 } Age (total effect) & 0.053 & 0.016 & .002 & -- \\
Age (direct effect) & 0.053 & 0.016 & .002 & -- \\
Cortisol Awakening Response (indirect effect) & -0.000 & 0.005 & -- & $-.011, .011$ \\
\hline
\end{tabular}
Model: $\mathrm{F}=8.4005, \mathrm{p}=.0007$, Adjusted $\mathrm{R}^{2}=.2120$

\section{Perceived Stress}

Analyses testing psychological stress as a mediator revealed a statistically significant positive relationship (total effect) between age and intrinsic motivation for physical activity ( $\beta$ $=.0371, \mathrm{SE}=.0155, \mathrm{p}=.0191$; see Table 3$)$. Age was negatively related to perceived stress $(B=-$ $0.3328, \mathrm{SE}=0.0974, \mathrm{p}=.0001)$. There was a positive relationship between perceived stress and intrinsic motivation $(\beta=-0.0305, \mathrm{SE}=$ 0.0184, $\mathrm{p}=.1019$ ). Bootstrap results (see Table 
3) revealed that the unique indirect effect through perceived stress was significant $(B=0.0103, \mathrm{SE}$ $=0.0061,95 \%$ CI: $0.0015,0.0267)$. The relationship between age and motivation, accounting for perceived stress (direct effect), was attenuated from the total effect (change in beta $=.0101)$ and became statistically non- significant $(\beta=.0270, \mathrm{SE}=.0165, \mathrm{p}=.1059)$; the ratio of the indirect effect to the total effect $\left(\mathrm{P}_{\mathrm{M}}\right)$ indicates that perceived stress accounts for approximately $28 \%$ of the total effect of age on intrinsic motivation for physical activity $\left(\mathrm{P}_{\mathrm{M}}=\right.$ .2776).

Table 3.

Indirect Effect of Perceived Stress in the Relationship between Age and Intrinsic Motivation for Physical Activity

\begin{tabular}{|c|c|c|c|c|}
\hline & \multicolumn{4}{|c|}{ Intrinsic Motivation for Physical Activity } \\
\hline & $\beta$ & $\mathrm{SE}$ & p-value & $95 \% \mathrm{CI}$ \\
\hline Age (total effect) & 0.037 & 0.016 & .019 & - \\
\hline Age (direct effect) & 0.027 & 0.017 & .106 & -- \\
\hline Perceived Stress (indirect effect) & 0.010 & 0.001 & -- & $.002, .027$ \\
\hline
\end{tabular}

\section{Associations with Physical Activity}

Notably, increasing age was significantly related to fewer minutes of moderate physical activity $(\mathrm{r}=-.308, \mathrm{p}=.012)$ as well as lower percent of time spent in moderate physical activity ( $\mathrm{r}=-.314$, $\mathrm{p}=.011)$. Intrinsic motivation for physical activity was not significantly related to minutes of moderate physical activity $(\mathrm{r}=-.09, \mathrm{p}=.486)$ or percent of time spent in moderate physical activity $(\mathrm{r}=-.102, \mathrm{p}=.425)$.

\section{Discussion}

We found that as age increased, pre-adolescent girls were more intrinsically motivated to engage in physical activity. Lower perceived stress was related to higher intrinsic motivation, however biological stress reactivity was not associated with intrinsic motivation to be physically active. Results testing the indirect effects of age through stress measures indicated perceived stress may be a pathway through which age could impact intrinsic motivation for physical activity; but the same was not true for our marker of physiological stress reactivity. Thus, psychological stress seems to impact motivation, but physiological stress reactivity, as measured by cortisol awakening response, may not. Given the crosssectional design of this study, true tests of the mediating effects of stress in longitudinal designs is warranted, as this may elucidate findings that increased age was related to less time in moderate physical activity. Of note, non-significant correlations between motivation and actual physical activity highlight that motivation may be necessary, but alone is not sufficient to guarantee being active.

As guided by the biopsychosocial model (Engel, 1977; Borrell-Carrió et al., 2004) and previous research (Wüst et al., 2000; Kudielka \& Wüst, 2010; Shirtcliff \& Ruttle, 2010), we expected that both perceived stress and cortisol awakening response would be pathways through which increased age may lead to lower motivation for physical activity. However, the non-significant association with physiological stress reactivity indicates that intrinsic motivation may be more affected by psychological state, rather than stress reactivity. The current data did not show a significant association between perceived stress and cortisol awakening response, implying that these measure distinct aspects of stress. Increased cortisol awakening response has been related to stressful events and anxiety (Chida \& Steptoe, 2009), while lower responses have been associated with depressive symptoms (Scheyer \& Urizar, 2016; Stetler \& Miller, 2005). In a metaanalysis, Dickerson and Kemeny (2004) concluded that cortisol may not be responsive to all stressors, but only those where social evaluation and/or lack of control are central.

While the use of objective, biological measures are of significant value, given the breadth of 
outcomes they may represent, they cannot replace the use of self-report measures, as directly asking people allows us to capture their unique, subjective experience of psychological distress.

It thus appears that the perception of stress could be a stronger influence on how increased age impacts intrinsic motivation for physical activity. While the specific associations tested in the current study may not have been previously explored, existing literature supports the importance of the influence of individual perception on physical activity and related outcomes. According to Crocker, Sabiston, and Kowalski (2006), longitudinal studies show physical self-perception was a strong predictor of physical activity among adolescent girls. Inchley, Kirby, and Currie (2011) reported that girls' physical self-perceptions greatly impacted their physical activity. Girls with lower perceived athletic competence have displayed a decreased enjoyment of physical education with an increasing age (Cairney, Kwan, \& Velduizen, 2012). Cox, Smith, and Williams (2008) reported that perceptions of competence, autonomy, and relatedness, self-determined motivation, enjoyment, and physical activity in physical education were positive predictors of leisure-time physical activity.

Contrary to Labbrozzi, Robazza, and Bertollo's (2013) findings, the current data indicated that intrinsic motivation increased with age among these minority girls; however, girls in our sample were the same age as the "younger girls" in Labbrozzi et al's study. Based on Tanner Stage, the current sample were all pre-pubertal. The oldest girls in the sample were 11.6 years old, so at this age, the pubertal decline in physical activity may not yet be as pronounced as those in later developmental stages. As anticipated, lower stress was related to higher intrinsic motivation. Thus, the enjoyment and pleasure associated with physical activity is likely less sustainable in the face of stress.

\section{Limitations}

Due to the small sample size, current analyses did not control for additional covariates so as not to further reduce power; future studies should adjust analyses for potential confounding variables, e.g., body image. The representativeness of the sample is also limited by the small sample; and findings are less generalizable to African American girls, considering their small numbers, and not generalizable to white samples. Cross-sectional analyses prohibit making any conclusions that mediation exists and that the pathway of association occurs in this direction, however, it presents a finding that may be worthy of additional exploration in longitudinal studies (the current study did not pursue longitudinal analyses due to small numbers at follow up).

Our measures also introduce other limitations. The low reliability for the intrinsic motivation scale was may have impacted the ability to detect associations as well as the estimates produced in analyses. The cortisol awakening response is just one indicator of physiological stress response, perhaps other biomarkers (e.g., catecholamine from the adrenal medulla (Dhabhar \& McEwen, 2001) or norepinephrine from nerve terminals (Webster, Tonelli, \& Sternberg, 2002) would have produced different results. Further, the operationalization of cortisol awakening response as an indicator of physiological stress reactivity is limited, given that the true function is yet unknown, and literature shows that it may be a marker of mental stress associated with preparing for the day's burden.

Our findings suggest that higher levels of perceived stress are related to lower levels of intrinsic motivation for exercise in prepubertal girls. Based on SDT, this may in turn result in less physical activity. Although the current data did not show a significant relationship between intrinsic motivation and physical activity, support for this association in previous studies (Owen et al., 2014) suggests that the complex relationships between intrinsic motivation and physical activity warrant further investigation. Results indicate that stressful environments for children may impact their motivation as they get older. This may be more pronounced in minority populations who face unique risk factors living in underserved communities.

\section{Conclusions}

Implications for Practice. Parents and teachers may be able to enhance physical activity among 
young girls by promoting stress reduction through teaching adaptive coping methods and providing supportive environments. For example, mindfulness training for stress reduction has been shown to positively impact mental health in elementary school children (van de WeijerBergsma, Langenberg, Brandsma, Oort, \& Bögels, 2014). A focus on positive psychology provides a promising prevention model that integrates the school psychologist (Terjesen, Jacofsky, Froh, \& DiGiuseppe, 2004). As girls traverse puberty, peer influence increases, so education that can enhance supportive peer networks may be effective in reducing stress that may have an impact on motivation to be physically active and engage in other healthful behaviors. Promoting positive peer networks through strategies to obtain peer social support and education on healthy relationships may prove useful. There is a need to continue to address these matters in schools and at home so young, minority girls hear positive messages around leading an active lifestyle and achieving emotional well-being.

Implications for Policy. Health-related programming in schools, such as physical education and health classes, tend to be the first to be reduced or cut due to budget constraints (Ennis, 2006; Lewin \& Dillon, 2010), which are more frequent in underserved communities. School district policies may better serve child health by protecting funding for these programs. Evidence shows that school-based programs to promote mental health can be effective on a wide scale (Wells, Barlow, \& Stewart-Brown, 2003). State policies that consistently allocate long-term funding towards school and community-based programs in economically disadvantaged areas that promote psychological well-being can help to address these issues for minority children.

Implications for Research. To enhance veracity of our current findings, replication is needed in studies that employ a longitudinal design with larger samples of children. Future studies should further examine the relationship of hormonal changes in young girls and their psychological states as these experiences can impact motivation to engage in healthy activities, which have longlasting effects into adulthood. Explorations of the moderating effect of pubertal stage on associations in datasets where the full range of Tanner stages are present are warranted.

\section{Acknowledgments}

Acknowledgement/Funding source: This work was supported by the National Institute of Diabetes and Digestive and Kidney Diseases (NIDDK), Grant \# KO1 DK59293-01.

\section{References}

Belcher, B. R., Berrigan, D., Dodd, K. W., Emken, B. A., Chou, C. P., \& Spuijt-Metz, D. (2010). Physical activity in US youth: Impact of race/ethnicity, age, gender, \& weight status. Medicine and Science in Sports and Exercise, 42(12), 2211.

Borrell-Carrió, F., Suchman, A. L., \& Epstein, R. M. (2004). The biopsychosocial model 25 years later: Principles, practice, and scientific inquiry. Annals of Family Medicine, 2(6), 576-582.

Cairney, J., Kwan, M. Y., Velduizen, S., Hay, J., Bray, S. R., \& Faught, B. E. (2012). Gender, perceived competence and the enjoyment of physical education in children: A longitudinal examination. International Journal of Behavioral Nutrition and Physical Activity, 9(1), 26.

Carrasco, G. A., \& Van de Kar, L. D. (2003). Neuroendocrine pharmacology of stress. European Journal of Pharmacology, 463(1-3), 235-272.

Centers for Disease Control and Prevention (CDC). (2018). CDC Youth Risk Behavior Surveillance United States, 2017. Morbidity Mortality Weekly Report, 2017, 67(8).

Chida, Y., \& Steptoe, A. (2009). Cortisol awakening response and psychosocial factors: A systematic review and meta-analysis. Biological Psychology, 80(3), 265-278.

Cohen, S., Janicki-Deverts, D., \& Miller, G. E. (2007). Psychological stress and disease. Journal of the American Medical Association, 298(14), 1685-1687. 
Cohen, S., Kamarck, T., \& Mermelstein, R. (1983). A global measure of perceived stress. Journal of Health and Social Behavior, 24(4), 385-396.

Cox, A. E., Smith, A. L., \& Williams, L. (2008). Change in physical education motivation and physical activity behavior during middle school. Journal of Adolescent Health, 43(5), 506-513.

Crocker, P. R., Sabiston, C. M., Kowalski, K. C., McDonough, M. H., \& Kowalski, N. (2006). Longitudinal assessment of the relationship between physical self-concept and health-related behavior and emotion in adolescent girls. Journal of Applied Sport Psychology, 18(3), 185-200.

Deci, E.L., \& Ryan, R.M. (1985). Intrinsic motivation and self-determination in human behavior. New York, NY: Plenum.

Dhabhar, F.S., \& McEwen, B.S. (2001). Bidirectional effects of stress and glucorticoid hormones on immune function: Possible explanations for paradoxical observations. In: R. Ader, D.L. Felten, \& N. Cohen (Ed.), Psychoneurimmunology (pp. 301-338).

Dickerson, S.S., \& Kemeny, M.E. (2004). Acute stressors and cortisol responses: A theoretical integration and synthesis of laboratory research. Psychological Bulletin, 130(3), 355.

Emmanuel, M., \& Bokor, B.R. (Updated May 13, 2019). Tanner Stages. In: StatPearls [Internet]. Retrieved from: https://www.ncbi.nlm.nih.gov/books/NBK470280/

Engel, G.L. (1977). The need for a new medical model: a challenge for biomedicine. Science, 196(4286), 129-36.

Ennis, C.D. (2006). Curriculum: Forming and reshaping the vision of physical education in a high need, low demand world of schools. Quest, 58(1), 41-59.

Foran, A. C., Cermak, S. A., \& Spruijt-Metz, D. (2013). Psychosocial determinants of participation in moderate-to-vigorous physical activity among Hispanic and Latina middle school-aged girls. Hispanic Health Care International, 11(3), 142-148.

Freedson, P.S., Melanson, E., \& Sirard, J. (1998). Calibration of the Computer Science and Applications, Inc. accelerometer. Medicine and Science in Sports and Exercise, 30(5), 777-781.

Fries, E., Dettenborn, L., \& Kirschbaum, C. (2009). The cortisol awakening response (CAR): Facts and future directions. International Journal of Psychophysiology, 72(1), 67-73.

Glanz, K., \& Schwartz, M. D. (2008). Stress, coping, and health behavior. Health behavior and health education: Theory, Research, and Practice, 4, 211-236.

Goudas, M., Biddle, S., \& Fox, K. (1994). Achievement goal orientations and intrinsic motivation in physical fitness testing with children. Pediatric Exercise Science, 6(2), 159-167.

Inchley, J., Kirby, J., \& Currie, C. (2011). Longitudinal changes in physical self-perceptions and associations with physical activity during adolescence. Pediatric Exercise Science, 23(2), 237-249.

Kudielka, B.M., \& Wüst, S. (2010). Human models in acute and chronic stress: Assessing determinants of individual hypothalamus-pituitary-adrenal axis activity and reactivity. Stress, 13(1), 1-14.

Labbrozzi, D., Robazza, C., \& Bertollo, M. (2013). Pubertal development, physical self-perception, and motivation toward physical activity in girls. Journal of Adolescence, 36(4), 759-765. https://doi.org/10.1016/j.adolescence.2013.06.002.

Lam, H.T. (2012). The effects of psychological stress, depressive symptoms, and cortisol on body mass and central adiposity in 10- to 12-year-old children (Doctoral dissertation, University of Alabama at Birmingham).

Lee, E.H. (2012). Review of the psychometric evidence of the perceived stress scale. Asian Nursing Research, 6(4), 121-127.

Levesque, C.S., Williams, G.C., \& Elliot, D. (2007). Validating the theoretical structure of the Treatment Self-Regulation Questionnaire (TSRQ) across three different health behaviors. Health Education Research, 22(5), 691-702.

Lewin, T., \& Dillon, S. (2010, April 20). Districts warn of deeper teacher cuts. The New York Times. Retrieved from: https://nytimes.com 
Lippke, S., Wienert, J., Kuhlmann, T., Fink, S., \& Hambrecht, R. (2015). Perceived stress, physical activity and motivation: Findings from an internet study. Annals of Sports Medicine and Research, 2(1).

National Center for Health Statistics. (2017). Health United States, 2016 With Chartbook on Trends in the Health of Americans.

Nguyen-Rodriguez, S.T., Unger, J.B., \& Spruijt-Metz, D. (2009). Psychological determinants of emotional eating in adolescence. Eating Disorders, 17(3), 211-224.

Ordaz, S., \& Luna, B. (2012). Sex differences in physiological reactivity to acute psychosocial stress in adolescence. Psychoneuroendocrinology, 37(8), 1135-1157. http://doi.org/10.1016/j.psyneuen.2012.01.002

Owen, K.B., Smith, J., \& Lubans, D.R. (2014). Self-determined motivation and physical activity in children and adolescents: A systematic review and meta-analysis. Preventive Medicine, 67, 270279.

Preacher, K.J., \& Hayes, A.F. (2008). Asymptotic and resampling strategies for assessing and comparing indirect effects in multiple mediator models. Behavior Research Methods, 40(3), 879891.

Preacher, K.J., \& Kelley, K. (2011). Effect size measures for mediation models: quantitative strategies for communicating indirect effects. Psychological Methods, 16(2):93-115.

Ryan, R.M., \& Deci, E.L. (2000). Self-determination theory and the facilitation of intrinsic motivation, social development, and well-being. American Psychologist, 55, 68-78.

Sanchez, H. H., Urizar, J. G., \& Yim, I. S. (2019). The influence of mindfulness and social support on stress reactivity during pregnancy. Stress and Health, 35(3), 330-340.

Scheyer, K., \& Urizar, G. G. (2016). Altered stress patterns and increased risk for postpartum depression among low-income pregnant women. Archives of Women's Mental Health, 19(2), 317328.

Shanahan, L., McHale, S. M., Osgood, D. W., \& Crouter, A. C. (2007). Conflict frequency with mothers and fathers from middle childhood to late adolescence: Within-and betweenfamilies comparisons. Developmental Psychology, 43(3), 539.

Shirtcliff, E.A., \& Ruttle, P. (2010). Immunological and neuroendocrine dysregulation following early deprivation and stress. In: K.H. Brisch (Ed.), Attachment and Early Disorders of Development. Klett-Cotta, Stuttgart, Munich.

Spruijt-Metz, D., Belcher, B. R., Hsu, Y. W., McClain, A. D., Chou, C. P., Nguyen-Rodriguez, S., Weigensberg, M.J., \& Goran, M. I. (2013). Temporal relationship between insulin sensitivity and the pubertal decline in physical activity in peripubertal Hispanic and African American females. Diabetes Care, 36(11), 3739-3745.

Spruijt-Metz, D., Nguyen-Michel, S. T., Goran, M. I., Chou, C. P., \& Huang, T. T. (2008). Reducing sedentary behavior in minority girls via a theory-based, tailored classroom media intervention. International Journal of Pediatric Obesity, 3(4), 240-248.

Stetler, C., \& Miller, G. E. (2005). Blunted cortisol response to awakening in mild to moderate depression: regulatory influences of sleep patterns and social contacts. Journal of Abnormal Psychology, 114(4), 697.

Terjesen, M. D., Jacofsky, M., Froh, J., \& DiGiuseppe, R. A. (2004). Integrating positive psychology into schools: Implications for practice. Psychology in the Schools, 41(1), 163-172.

Tremblay, L., \& Frigon, J. Y. (2005). The interaction role of obesity and pubertal timing on the psychosocial adjustment of adolescent girls: Longitudinal data. International Journal of Obesity, 29(10), 1204.

Tsigos, C., \& Chrousos, G. P. (2002). Hypothalamic-pituitary-adrenal axis, neuroendocrine factors and stress. Journal of Psychosomatic Research, 53(4), 865-871.

U.S. Department of Health and Human Services. (2018). Physical Activity Guidelines Advisory Committee report. Washington, DC: U.S. Department of Health and Human Services. 
van de Weijer-Bergsma, E., Langenberg, G., Brandsma, R., Oort, F. J., \& Bögels, S. M. (2014). The effectiveness of a school-based mindfulness training as a program to prevent stress in elementary school children. Mindfulness, 5(3), 238-248.

Wang, C. J., \& Biddle, S. J. (2001). Young people's motivational profiles in physical activity: A cluster analysis. Journal of Sport and Exercise Psychology, 23(1), 1-22.

Webster, J. I., Tonelli, L., \& Sternberg, E. M. (2002). Neuroendocrine regulation of immunity. Annual Review of Immunology, 20(1), 125-163.

Wells, J., Barlow, J., \& Stewart-Brown, S. (2003). A systematic review of universal approaches to mental health promotion in schools. Health Education, 103(4), 197-220.

Wilhelm, I., Born, J., Kudielka, B. M., Schlotz, W., \& Wüst, S. (2007). Is the cortisol awakening rise a response to awakening?. Psychoneuroendocrinology, 32(4), 358-366.

Wüst, S., Federenko, I., Hellhammer, D. H., \& Kirschbaum, C. (2000). Genetic factors, perceived chronic stress, and the free cortisol response to awakening. Psychoneuroendocrinology, 25(7), 707-720.

Yli-Piipari, S., Watt, A.P., \& Jaakkola, T. (2009). Relationships between physical education students' motivational profiles, enjoyment, state anxiety, and self-reported physical activity. Journal of Sports Science and Medicine, 8, 327-336.

Corresponding Author Information

Selena Nguyen-Rodriguez (ORCID: 0000-0002-7418-5752)

Department of Health Science, Health \& Human Services

Bldg. 2 (HHS-2), College of Health and Human Services, California State University, Long Beach, CA 90840

email: selena.nguyen-rodriguez@csulb.edu

phone: 562-985-4111 